\title{
Panorama Brasileiro de Uso de Ferramentas para Desenvolvimento do Pensamento Computacional e Ensino de Programação
}

\author{
Carmen Vera Scorsatto Brezolin ${ }^{1,2}$, Milene Selbach Silveira ${ }^{1}$ \\ ${ }^{1}$ Escola Politécnica - Pontifícia Universidade Católica do Rio Grande do Sul (PUCRS) \\ Avenida Ipiranga 6681 - Porto Alegre - RS - Brasil \\ ${ }^{2}$ Instituto Federal Sul Riograndense - Campus Passo Fundo (IFSUL) \\ Estrada Perimetral Leste, 150 - Passo Fundo - RS - Brasil \\ carmen.brezolin@edu.pucrs.br, milene.silveira.apucrs.br
}

\begin{abstract}
Resumo. Uma das abordagens que tem sido utilizadas para apoiar o aprendizado de algoritmos e programação e o desenvolvimento do pensamento computacional é o uso de ferramentas tecnológicas projetadas para este fim. No intuito de se compreender o cenário brasileiro relacionado a este uso, foi realizado um mapeamento sistemático, nos principais fóruns brasileiros, promovidos pela Sociedade Brasileira de Computação que discutem o tema (tais como o SBIE, WIE, WEI e IHC, dentre outros). Com a análise realizada, verificou-se que o número maior de artigos é direcionado ao Ensino Fundamental e que as ferramentas mais utilizadas foram as Linguagens de Programação Visual com Blocos, com destaque para o Scratch. Ressalta-se a necessidade de se levar a pesquisa aos níveis técnicos e infantil e de um envolvimento maior dos professores nestas ações.
\end{abstract}

Abstract. One of the approaches that has been used to support the learning of algorithms and programming and the development of computational thinking is the use of technological tools designed for this purpose. In order to understand the Brazilian scenario related to this use, a systematic mapping was carried out in the main Brazilian forums, promoted by the Brazilian Computer Society, that discuss the topic (such as SBIE, WIE, WEI and IHC, among others). With the analysis performed, it was found that the largest number of articles is directed to elementary education and that the most used tools were the Visual Programming Languages with Blocks, with emphasis on Scratch. It is emphasized the need to take the research to the other educational levels and also the need of a greater involvement of teachers in these actions.

\section{Introdução}

Disciplinas de Algoritmos e Introdução à Programação tendem a gerar traumas nos alunos iniciantes, ocasionando altos níveis de desistências nos primeiros anos dos cursos [Medida e Fertig 2006]. Amaral, Medina e Tarouco (2016) afirmam que é possível perceber a dificuldade no desenvolvimento de competências que abrangem, de forma geral, a capacidade de utilização de recursos computacionais e estratégias algorítmicas para a resolução de problemas, por meio da abstração e decomposição lógica desses problemas.

Estas competências estão relacionadas ao conceito de Pensamento Computacional (PC). Este conceito, introduzido por Wing (2006), envolve a resolução de problemas, o 
projeto de sistemas e o entendimento do comportamento humano, baseando-se nos conceitos fundamentais da Ciência da Computação. Ainda para Wing (2014), o Pensamento Computacional descreve a atividade mental na formulação de um problema para admitir uma solução computacional. Além disso, ele não é apenas sobre a resolução de problemas, mas também sobre a formulação desses problemas [Wing 2014]. PC é definido como uma forma de "desenvolver e empregar estratégias para entender e resolver problemas de forma a aproveitar o poder dos métodos tecnológicos para desenvolver e testar soluções" [ISTE 2016]. Em uma revisão da literatura sobre o tema, no contexto brasileiro, Zanetti, Borges e Ricarti (2016) afirmam que habilidades como coleta, análise e representação de dados, decomposição de problemas, abstração e desenvolvimento de algoritmos encontram-se na maioria dos artigos analisados.

Uma das abordagens que tem sido utilizadas - tanto para apoiar alunos de Ensino Superior que estão começando a trabalhar com estes conceitos quanto para desenvolver o pensamento computacional em alunos da Educação Básica - é o uso de ferramentas tecnológicas projetadas para este fim. Assim, para compreender o cenário brasileiro relacionado a este uso, foi feito um mapeamento sistemático, nos principais fóruns brasileiros, promovidos pela Sociedade Brasileira de Computação (SBC) que discutem o tema (tais como o SBIE, WIE, WEI e IHC, dentre outros).

A fim de apresentar a pesquisa realizada, o artigo está organizado da seguinte forma: a Seção 2 apresenta trabalhos relacionados; a Seção 3, conceitos de Pensamento Computacional; a Seção 4, a metodologia seguida; a Seção 5, uma análise geral dos resultados e discussões; e, por fim, a Seção 6, as considerações finais.

\section{Trabalhos Relacionados}

Ao se iniciarem as pesquisas sobre este tema, foram encontradas outras revisões da literatura como por exemplo, o mapeamento de Avila et al. (2016), que teve como objetivo descrever os desdobramentos, no Brasil, sobre o tema de Pensamento Computacional, identificando o público-alvo, número de participantes, instrumentos ou ambientes adotados. Os autores ressaltam que as experiências de ensino são, na sua maioria, aplicadas ao Ensino Fundamental, utilizando as ferramentas Scratch e Computacão Desplugada. Relatam ainda observar nos participantes um aumento do interesse pela área da Computação.

Já o estudo de Zabot, Andrade e Matos (2018) tinha o objetivo de identificar como os jogos digitais podem ser usados para o desenvolvimento das habilidades do raciocínio computacional. O trabalho identificou as principais mecânicas de jogo, os gêneros mais adequados e as principais habilidades desenvolvidas no raciocínio computacional, tanto no ato de jogar, quanto no ato de criação do artefato de jogo.

Nascimento, Santos e Tanzi (2018), em seu mapeamento, procuraram identificar artigos que abordassem o Pensamento Computacional de maneira que este contribuísse para a aprendizagem de disciplinas da educação básica. Os autores destacam que a maioria dos artigos selecionados apresentava iniciativas voltada para o Ensino Fundamental II, concluem que um passo importante seria o reconhecimento da Computação enquanto ciência na educação básica.

Por fim, o mapeamento de Ortiz e Pereira (2018), realizado em uma base nacional e três bases internacionais, buscou verificar quais países mais pesquisam este tema. $\mathrm{O}$ estudo demonstrou que os Estados Unidos possuem maior representatividade, seguido 
pelo Brasil. O estudo observou, também, que os Estados Unidos abordam uma maior diversidade de públicos, apresentando pesquisas em todos os níveis, e o Brasil realizou todas as iniciativas na educação básica. Sobre as ferramentas utilizadas nas atividades, as mais citadas foram a Computação Desplugada, o uso do Scratch e de kits de robótica.

Os mapeamentos mencionados são relevantes no tocante ao uso do Pensamento Computacional, no entanto, eles não possuem enfoque maior nas ferramentas utilizadas neste contexto, foco principal do trabalho aqui apresentado.

\section{Pensamento Computacional}

Para Guarda e Pinto (2020), o Pensamento Computacional se tornou um tópico de pesquisa de interesse do mundo, com vários aspectos para serem investigados, incluindo diferentes faixas etárias, segmentos educacionais, bem como ferramentas para o desenvolvimento e instrumentos para avaliá-lo.

Em publicação da ISTE (2016), o Pensamento Computacional é definido como uma forma de "desenvolver e empregar estratégias para entender e resolver problemas de forma a aproveitar o poder dos métodos tecnológicos para desenvolver e testar soluções". Papert (1985) usava o termo "pensamento de procedimento" e afirmava que era necessário promover esse pensamento junto aos alunos, criando condições para as crianças usarem um pensamento de procedimento de forma efetiva e divertida, pois todo mundo trabalha com procedimentos na vida diária, mas esses são vividos e usados, não são necessariamente objeto de reflexão.

Existem três pilares básicos que fundamentam o Pensamento Computacional segundo Andrade et al. (2013): a abstração, a automação e a análise. A abstração, para os autores, é a capacidade de extrair apenas as características importantes de um problema para chegar à sua solução, levando em consideração que as demais já foram solucionadas; a automação é a utilização de um meio eletrônico na substituição do trabalho manual; e, por fim, a análise é o estudo dos resultados gerados pela automação.

Os conceitos que fundamentam o Pensamento Computacional aproximam-se dos conceitos sobre resolução de problemas da matemática apresentado por Polya (1995). Esse autor apresenta quatro fases como sugestão de como resolver um problema: compreender o problema, verificar como os dados estão inter-relacionados para estabelecer um plano, executar o plano e realizar o retrospecto da resolução.

Por fim, o pensamento computacional não é só ou tudo sobre Ciência da Computação. Segundo Brackmann (2017), os benefícios educacionais de poder pensar computacionalmente, começando com o uso de abstrações, aprimoram e reforçam as habilidades intelectuais e, portanto, podem ser transferidos para qualquer domínio. A ciência e a sociedade se beneficiarão das descobertas e inovações produzidas por uma força de trabalho treinada para pensar computacionalmente [Brackmann 2017].

\section{Metodologia}

Para a concretização deste estudo, realizou-se um Mapeamento Sistemático, seguindo as diretrizes apresentadas Kitchenham e Charters (2007). As seções a seguir apresentam este planejamento em detalhe. 


\subsection{Questões de pesquisa}

Com o objetivo de investigar o panorama brasileiro de uso de ferramentas tecnológicas para apoio ao desenvolvimento de pensamento computacional e ensino de programação, foi delineada a seguinte questão principal de pesquisa: Qual o estado da arte referente às ferramentas tecnológicas utilizadas para promover o pensamento computacional e o ensino de programação?

E, a fim de apoiar a análise da questão de pesquisa principal, foram estabelecidas as seguintes questões de pesquisa secundárias: QP1 - Qual o público-alvo destes estudos? QP2 - Qual o objetivo principal destes estudos? QP3 - Quais ferramentas têm sido utilizadas?

\subsection{Bases de Dados e Estratégias da Pesquisa}

Para obtenção das publicações, foi realizada a busca de artigos completos, que estão disponibilizados no formato digital, acessíveis via Internet. Os fóruns e bases estão descritos a Tabela 1. O período selecionado para análise dos artigos foi dos últimos 15 anos, desde 2006, data em que Wing (2006) introduziu o termo Pensamento Computacional. Ainda devido à indisponibilidade digital dos anais de todas as edições dos Workshops do CBIE, do WAlgProg e do WEI, não foi possível obter um recorte preciso destes eventos dos últimos quinze anos. O SBIE, o WAlgProg e WIE são eventos paralelos que ocorrem no CBIE, os WCBIE são os artigos incluídos nos Workshops do CBIE.

Tabela 1. Fóruns Selecionados

\begin{tabular}{|c|c|c|c|}
\hline Fórum & Descrição & Base $^{1}$ & Período \\
\hline SBIE & Simpósio Brasileiro de Informática na Educação & \multirow[t]{5}{*}{ CE-IE } & $2006-2020$ \\
\hline WIE & Workshop de Informática na Escola & & $2006-2020$ \\
\hline WCBIE & Workshops do Congresso Brasileiro de Informática na Educação & & $2012-2020$ \\
\hline WAlgProg & $\begin{array}{l}\text { Workshop de Ensino em Pensamento Computacional, } \\
\text { Algoritmos e Programação }\end{array}$ & & $2015-2020$ \\
\hline RBIE & Revista Brasileira de Informática na Educação & & $2006-2020$ \\
\hline WEI & Workshop sobre Educação em Computação & SBC & $2014-2020$ \\
\hline IHC & $\begin{array}{l}\text { Simpósio Brasileiro sobre Fatores Humanos em Sistemas } \\
\text { Computacionais }\end{array}$ & $\mathrm{ACM}$ & $2006-2020$ \\
\hline
\end{tabular}

A nomenclatura para denominação de Pensamento Computacional e ensino de programação é muito heterogênea, sendo que alguns autores utilizam, como sinônimos, termos como Raciocínio Computacional, Aprendizagem de Programação, Lógica de Programação, dentre outros, que também podem ter outros significados. Desta forma, optou-se pelo uso de diferentes termos de busca, conforme apresentado na Tabela 2.

Tabela 2. Termos de Busca

\begin{tabular}{|l|l|}
\hline Termo Principal & Termos Alternativos \\
\hline Pensamento Computacional & Computational thinking - Raciocínio Computacional \\
\hline \multirow{5}{*}{ Ensino de Programação } & Aprendizado de Programação - Aprendizagem de programação \\
& Aprender programação de computadores - Algoritmos e Programação \\
& - Conceitos de programação - Ensino de Algoritmos- Ensinar \\
& Programação - Ensino-aprendizagem de programação - \\
& Aprendizagem de Lógica e Programação - Introdução à programação \\
& - Programação de computadores - Ensino de lógica de programação - \\
& Lógica de Programação \\
\hline
\end{tabular}

\footnotetext{
${ }^{1}$ CEIE - https://www.br-ie.org/pub/ = SBC - https://sol.sbc.org.br/ = ACM - https://dl.acm.org/
} 
Inicialmente foi pesquisado, em cada fórum, se o título e resumo do artigo continham as palavras citadas na Tabela 2. Após, foi feita uma filtragem manual pelos termos principais e suas variações. Essa estratégia foi utilizada para filtrar possíveis modificações desses termos, para ter acesso ao maior número possível de artigos. Para realizar a seleção dos artigos foram utilizados os critérios de inclusão e exclusão destacados na Tabela 3.

Tabela 3. Critérios de inclusão e exclusão

\begin{tabular}{|c|l|}
\hline \multirow{4}{*}{ Critérios de inclusão } & Temas \\
\hline \multirow{4}{*}{$\begin{array}{c}\text { Critérios de exclusão } \\
\text { em títulos e resumos }\end{array}$} & Artigos completos \\
\cline { 2 - 2 } & Palavras que contemplem os termos de busca \\
\cline { 2 - 2 } & Publicações no período de 2006 até 2020 \\
\cline { 2 - 2 } & Artigos resumidos \\
\cline { 2 - 2 } & Artigos duplicados que tratam de questões políticas \\
\cline { 2 - 2 } & Artigos que tratam de infraestrutura dos laboratórios \\
\cline { 2 - 2 } $\begin{array}{c}\text { Critérios de exclusão no } \\
\text { texto completo }\end{array}$ & Artigos que tratam da formação de professores \\
\cline { 2 - 2 } & Artigos que tratam de Computação Desplugada \\
\cline { 2 - 2 } & Artigos que tratam de Análise de Projetos Pedagógicos de Cursos \\
\cline { 2 - 2 } & Artigos que tratam de Ensino de Estruturas de Dados \\
\cline { 2 - 2 } & Artigos que apresentam Mapeamentos Sistemáticos sobre o tema ${ }^{2}$ \\
\hline
\end{tabular}

O processo de pesquisa foi realizado seguindo as estratégias descritas e os números de artigos resultante em cada processo $^{3}$ são apresentados na Tabela 4 . Como primeiros resultados após aplicação da string de busca formada pelos termos da Tabela 3, foram selecionados 839 artigos. Com a aplicação dos primeiros critérios de exclusão, analisando-se títulos e resumos, foram identificadas 388 publicações. Na terceira etapa, com a leitura completa, e após os critérios de exclusão chegou-se a um conjunto final de $234 \operatorname{artigos}^{4}$ para análise.

Tabela 4. Resultados do Processo de Pesquisa

\begin{tabular}{|l|c|c|c|}
\hline \multirow{2}{*}{ Eventos } & \multicolumn{3}{|c|}{ Número de artigos } \\
\cline { 2 - 4 } & $\begin{array}{c}\text { Aplicação da } \\
\text { string de busca }\end{array}$ & $\begin{array}{c}\text { Aplicação dos Critérios de } \\
\text { Exclusão (títulos e resumos) }\end{array}$ & $\begin{array}{c}\text { Aplicação dos Critérios de } \\
\text { Exclusão (texto completo) }\end{array}$ \\
\hline WIE & 208 & 99 & 76 \\
\hline SBIE & 209 & 103 & 43 \\
\hline WAIgProg & 153 & 57 & 41 \\
\hline WCBIE & 74 & 34 & 43 \\
\hline WEI & 119 & 62 & 16 \\
\hline RBIE & 35 & 15 & 8 \\
\hline IHC & 41 & 18 & $\mathbf{2 3 4}$ \\
\hline Total: & $\mathbf{8 3 9}$ & $\mathbf{3 8 8}$ & \\
\hline
\end{tabular}

\subsection{Classificação dos artigos}

Durante a leitura foi possível classificar os artigos agrupando-os em categorias correspondentes as questões de pesquisa do mapeamento sistemático. O esquema de

\footnotetext{
${ }^{2}$ Mapeamentos sistemáticos, mesmo excluídos desta fase, foram utilizados como trabalhos relacionados

${ }^{3}$ As etapas podem ser consultadas em http://tiny.cc/dw8vtz

${ }^{4}$ As referências podem ser consultadas em https://tinyurl.com/yday9k38
} 
classificação estabelecido é apresentado na Tabela 5. Destaca-se que, ao classificar os artigos de acordo com as ferramentas utilizadas, observamos que junto das Linguagem de Programação Visual, existem as que mesclam imagens e/ou componentes visuais, as que utilizam blocos de encaixes e as que usam de gráficos estilo UML, assim foram criadas três categorias para melhor classificá-las.

Tabela 5. Categorias correspondentes as questões de pesquisa

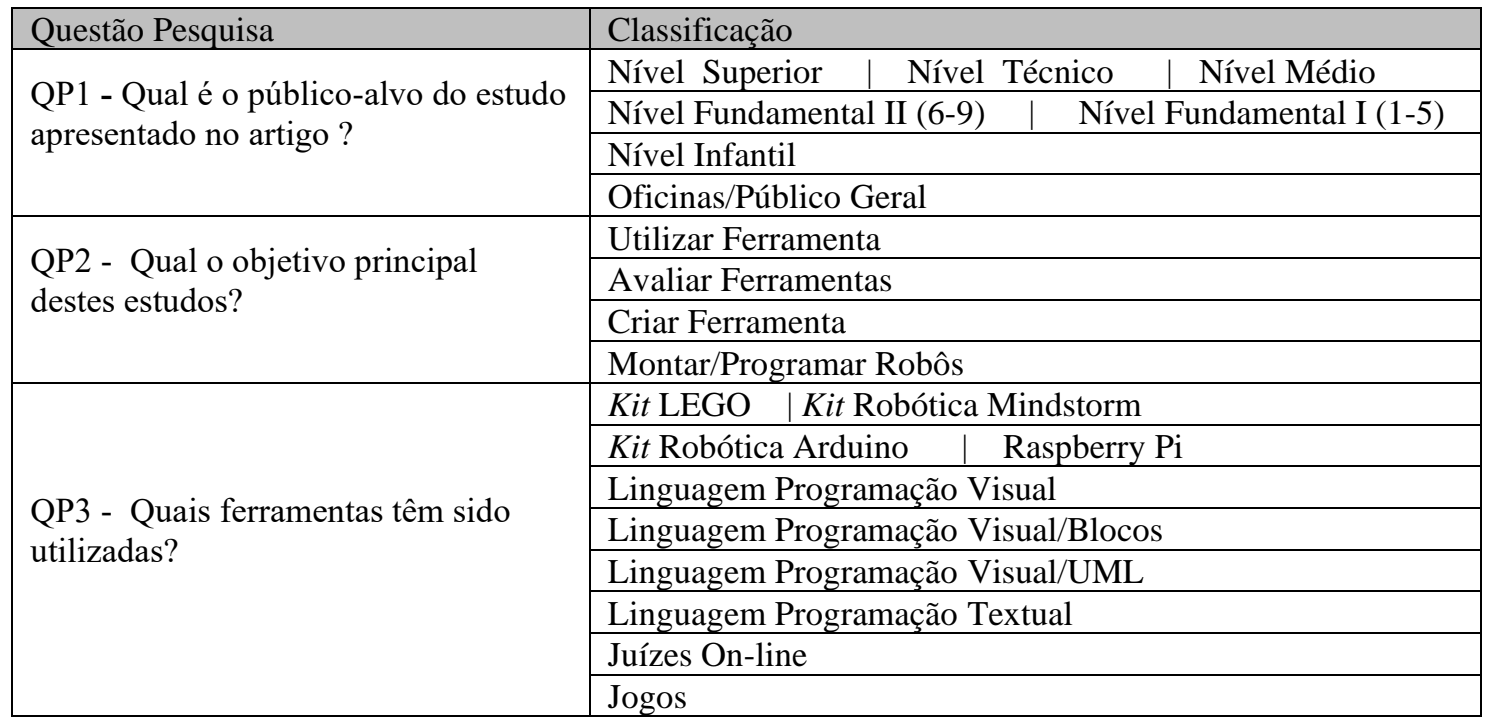

\section{Resultados e Discussão}

Uma das questões que motivou esse mapeamento sistemático foi observar para que público-alvo estão sendo direcionados os estudos publicados no Brasil, que se referem ao uso de ferramentas tecnológicas para promover o Pensamento Computacional e o ensino de programação. Como ilustrado na Figura 5a, o número maior de artigos é direcionado ao Ensino Fundamental: do total de 234 artigos, 61 são para o Nível Fundamental II, 43 ao Nível Fundamental I, totalizando 104 artigos, ou seja 44,5\%, direcionados a este nível de ensino. Os níveis Superior e Médio ocupam o segundo e terceiro lugares em número de artigos, sendo que os níveis que menos possuem atenção são os níveis Técnicos, Infantil e os direcionados ao público em Geral (Figura 5b).

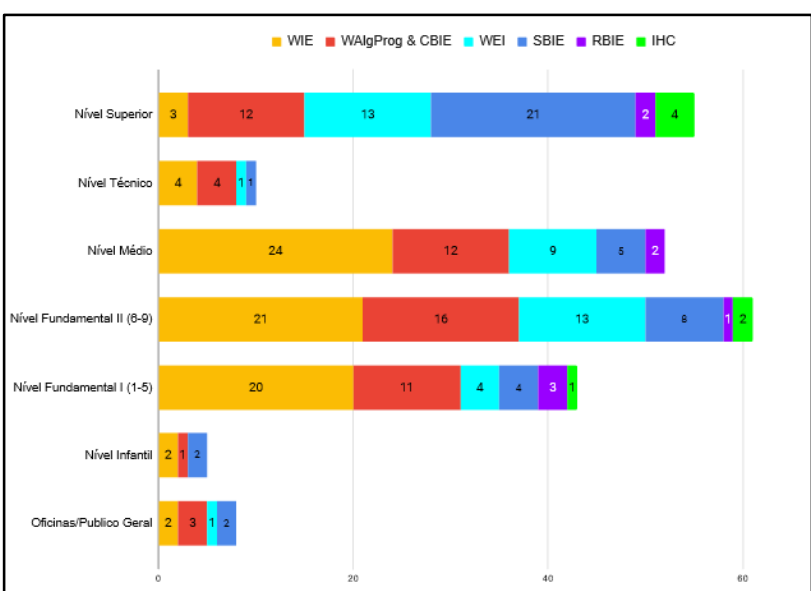

(a)

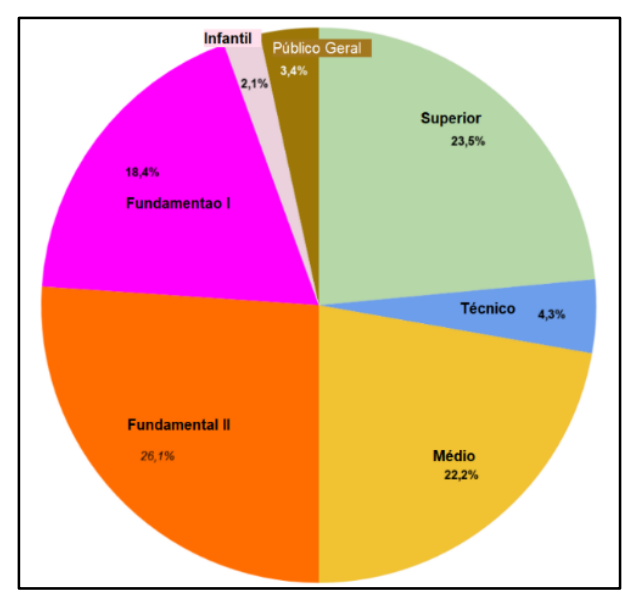

(b)

Figura 5. Público-alvo 
Um dos interesses ressaltado nesses artigos é o de fomentar e estimular os alunos ao conhecimento dos assuntos tratados em Ciência da Computação. Com o propósito de divulgar os cursos das áreas de tecnologia, na maioria das vezes, alunos vão até as escolas executar seus projetos universitários. E, assim como ilustrado no gráfico da Figura 6, os níveis Fundamental II, Médio e Superior ocupam a maioria dos estudos $(71,8 \%)$.

Quanto ao objetivo principal destes estudos, observou-se que a maioria (137 de 234) se concentrou em utilizar uma ferramenta tecnológica ou jogo, com o objetivo de promover o pensamento computacional e o ensino de programação (Figura 7).

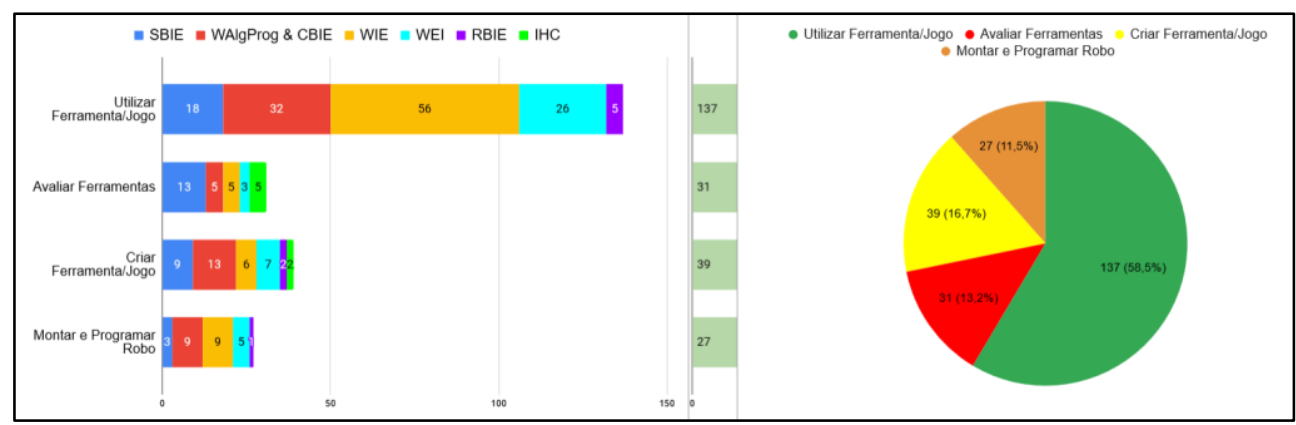

Figura 7. Objetivos por Fórum

Outro ponto que emergiu dos dados foi que os estudos que possuem como objetivo avaliar ferramentas representam 13,2 \% dos artigos, sendo que a maioria deles $(90,6 \%)$ fazem algum tipo de validação por experimento.

Em relação às ferramentas utilizadas, a maioria dos estudos utiliza Linguagens de Programação Visual com Blocos. 102 artigos utilizaram esse tipo de ferramenta, conforme evidenciado na Figura 8 (L P Blocos). Os jogos ocupam o segundo lugar, seguidos das linguagens de programação textuais e visuais e dos kits de Robótica Mindstorms e Arduíno.

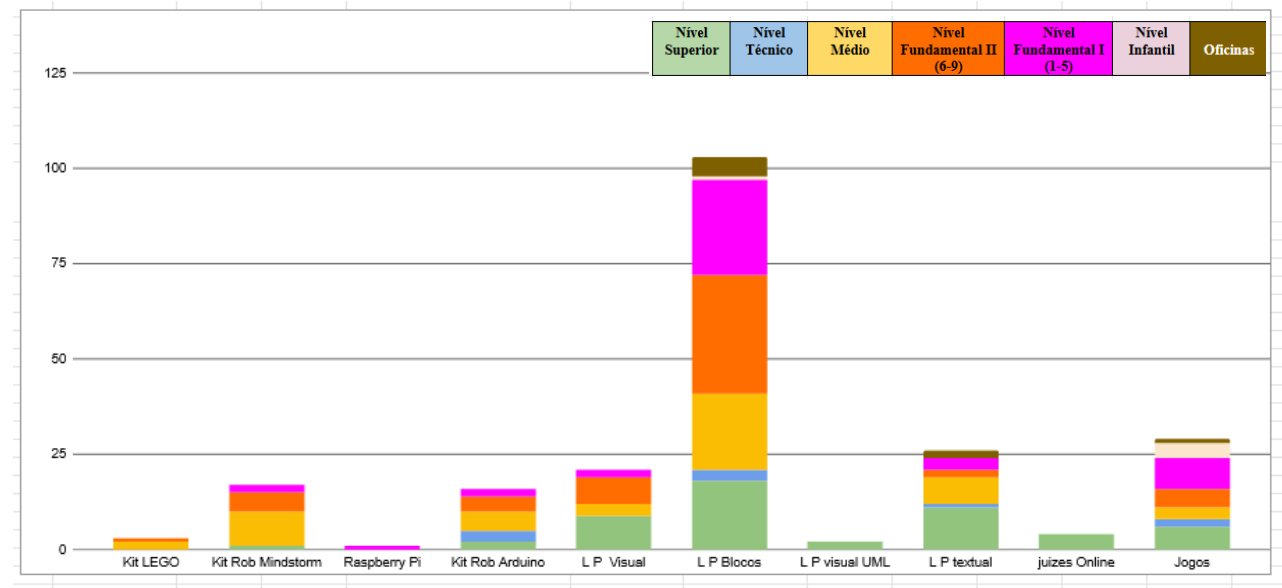

Figura 8. Categorias de Ferramentas

Os artigos que abordaram Linguagens de Programação em Blocos, apresentaram uma grande variedade de opções de ferramentas, em torno de 30 tipos diferentes, conforme Tabela 6. A ferramenta mais citada foi o Scratch (67 vezes), seguida pela plataforma do cod.org (23 vezes) e pelo App Inventor (15 vezes). 
Tabela 6. Ferramentas por Nível ${ }^{5}$

\begin{tabular}{|c|c|c|c|c|c|c|c|}
\hline Nome & Superior & Técnico & Médio & $\begin{array}{c}\text { Fundamental } \\
\text { II (6-9 ano) }\end{array}$ & $\begin{array}{c}\text { Fundamental } \\
\text { I (1-5 ano })\end{array}$ & Infantil & Oficinas \\
\hline LP Visual & \begin{tabular}{|l}
-iVProg (3) \\
-Alice \\
-JavaTool \\
-Logo \\
-Game Logic \\
-Múltipla \\
-Visual \\
$\quad$ Programmer \\
\end{tabular} & & $\begin{array}{l}\text {-Minecraft } \\
\text {-PROGLIB } \\
\text {-Visualg } \\
\text {-iVprog }\end{array}$ & $\begin{array}{l}\text {-Newprog+ } \\
\text {-EANewProg } \\
\text {-PhET } \\
\text {-Unit3D } \\
\text {-Logo (2) }\end{array}$ & $\begin{array}{l}\text {-NewProg, } \\
\text {-Logo }\end{array}$ & & \\
\hline $\begin{array}{l}\text { LP Blocos/ } \\
\text { Visual }\end{array}$ & $\begin{array}{l}\text {-Scratch (13) } \\
\text {-App Inventor(2) } \\
\text {-Python } \\
\quad \text { HelpBlock } \\
\text {-Ardublock } \\
\text {-Minibloq } \\
\text {-ModKit Micro } \\
\text {-Physical Etoys } \\
\text {-S4A } \\
\text {-LindenScripting } \\
\quad \text { Language } \\
\text {-LightBot } \\
\text {-Plataforma } \\
\quad \text { LERO } \\
\text {-Code.org (2) } \\
\text {-AgentSheet } \\
\text {-Blockly } \\
\text {-KTurtle }\end{array}$ & $\begin{array}{l}\text {-S4A } \\
\text {-Scratch (2) }\end{array}$ & $\begin{array}{l}\text { - Scratch (13) } \\
\text { - App Inventor } \\
\text { (7) } \\
\text { - Coding Dojo } \\
\text { (Blockly) } \\
\text { - Pinte o } 7 \\
\text { - S4A (2) } \\
\text { - Code.org } \\
\text { - Construct } 2 \\
\text { - iVProg } \\
\text { - Stencyl }\end{array}$ & $\begin{array}{l}\text {-Scratch (25) } \\
\text {-Code.org (9) } \\
\text {-Stencyl } \\
\text {-S4A } \\
\text {-Silent Teacher } \\
\text {-Poredu } \\
\text {-App } \\
\quad \text { Inventor (4) } \\
\text {-Blockly Games } \\
\text {-AgentSheet }\end{array}$ & $\begin{array}{l}\text {-Scratch (11) } \\
\text {-Code.org (8) } \\
\text {-Blockly (2) } \\
\text {-Monster Coding } \\
\text { (2) } \\
\text {-CodeMonkey (2) } \\
\text {-Pencil Code (2) } \\
\text {-Tynker Project } \\
\text {-Code Avengers } \\
\text {-Robô no } \\
\text { Labirinto } \\
\text {-ZerobotAPP } \\
\text {-AppInventor }\end{array}$ & $\begin{array}{l}\text { Scratch Jr } \\
\text { Code } \\
\text { Monkey } \\
\text { Tynker } \\
\text { Project }\end{array}$ & $\begin{array}{l}\text {-Blockly } \\
\text {-Scratch(4) } \\
\text {-Code.org } \\
\text { (3) } \\
\text {-Code } \\
\text { Monkey } \\
\text {-Monster } \\
\text { Coding } \\
\text {-App } \\
\text { Inventor }\end{array}$ \\
\hline LP Textual & $\begin{array}{l}\text {-LaraApp } \\
\text {-Analisador Facil } \\
\text {-Portugol Studio } \\
\text {-Python (2) } \\
\text {-BRc } \\
\text {-PortuCol } \\
\text {-Javascript }\end{array}$ & $\begin{array}{l}-\mathrm{AmPaRe} \\
-\mathrm{C}\end{array}$ & $\begin{array}{l}\text {-Python (2) } \\
\text {-IDE Portugol } \\
\text {-IDE Bipide } \\
\text {-CodeBlocks: } \\
\text {-Studio } \\
\text { Sketchpad } \\
\text {-VPL (Virtual } \\
\text { Programming } \\
\text { Lab } \\
\text {-IDE Portugol } \\
\text { Studio } \\
\end{array}$ & -Python (2) & $\begin{array}{l}\text {-IDE Portugol } \\
\text {-Linguagem NXC }\end{array}$ & & $\begin{array}{l}\text {-Python } \\
\text {-Visualg }\end{array}$ \\
\hline Jogos & $\begin{array}{l}\text {-Hanoi Tower } \\
\text {-Crossing Code } \\
\text {-Boolamp } \\
\text {-Invade the } \\
\text { System } \\
\text {-Code Dungeon } \\
\text {-Caça Bugs } \\
\text {-CodeCombat } \\
\text {-CodeSchool } \\
\text {-Klouro } \\
\text {-SORTIA } \\
\text {-Escracho } \\
\text {-Game Maker } \\
\text { - NoBug's Snack }\end{array}$ & \begin{tabular}{|l}
-Ninja \\
Prog \\
-DOTA 2
\end{tabular} & $\begin{array}{l}\text {-LightBot (4) } \\
\text {-CodeCombat } \\
\text {-NoBug's } \\
\text { Snack Bar } \\
\text {-RoboMind } \\
\text {-Robocode }\end{array}$ & $\begin{array}{l}\text {-Caça ao } \\
\text { Tesouro } \\
\text {-Bomberman } \\
\text {-CodeCombat } \\
\text {-Lightbot (2) } \\
\text { - WAlgor } \\
\text { - Classcraft } \\
\text { - BloxorZ } \\
\text {-MineCraft } \\
\text {-Portugol } \\
\text { Studio }\end{array}$ & $\begin{array}{l}\text {-A última árvore } \\
\text {-LightBot } \\
\text {-Furbot Móvel } \\
\text {-Furbot } \\
\text {-EcoLogic } \\
\text {-The Foos } \\
\text {-Play Code Dog } \\
\text {-Robotizen } \\
\text {-RUN Marco } \\
\text {-CodeCombat (2) } \\
\text {-Minecraft }\end{array}$ & $\begin{array}{l}\text {-Code } \\
\text { Baymax } \\
\text {-Code } \\
\text { Monkey } \\
\text {-Code Studio } \\
\text {-Lightbot } \\
\text {-Ninja Shape } \\
\text {-The Foos (3) } \\
\text {-Code Studio } \\
\text {-Run Marco } \\
\text {-Tynker } \\
\text {-Robot } \\
\text { School }\end{array}$ & -Lightbot \\
\hline \begin{tabular}{|l} 
Kit \\
LEGO
\end{tabular} & & & $\begin{array}{l}\text {-Kit LEGO, } \\
\text {-Oeco Tech, } \\
\text {-Robo TX - } \\
\text { Training lab }\end{array}$ & -Robo MBot & & & \\
\hline \begin{tabular}{|l} 
Kit Robótica \\
Mindstorm
\end{tabular} & -RoboMind & & Kit Robótica(8) & \begin{tabular}{|l}
-Kit \\
Robótica(4) \\
\end{tabular} & -Scratch S4 (3) & & \\
\hline $\begin{array}{l}\text { Kit Robótica } \\
\text { Arduino }\end{array}$ & -RoboMind (2) & -Arduino & $\begin{array}{l}\text {-Robo Sumo } \\
\text { UR2 } \\
\text {-Arduino (2) } \\
\text {-RoboCode } \\
\text {-Tonkercad }\end{array}$ & -Arduino (4) & -DuinoBlock(2) & & \\
\hline
\end{tabular}

${ }^{5}(\mathrm{x})$ - O número entre parênteses do lado do nome da ferramenta é o número de vezes que essa foi citada entre os artigos. 
As categorias menos citadas foram a de computador de baixo custo, com o Raspberry Pi citado uma vez no nível Fundamental I; a de Linguagem de Programação Visual com UML, duas vezes relacionada ao ensino superior; e, a categoria Juízes Online, também utilizados nos cursos superiores, com as ferramentas: MOJO, PROGTEST e CodeBench (2). Pelo baixo índice de uso, essas três categorias foram suprimidas da Tabela 6.

Por fim, observamos que o Scratch é uma ferramenta que é utilizada em todos os níveis, do Infantil até o Superior, e a plataforma do code.org é utilizada mais nos níveis Fundamental I, Fundamental II e Médio. A categorias de jogos também apresentou uma lista com cerca de 32 nomes diferentes, sendo os mais citados o Lightbot ( 9 vezes), o CodCombat ( 5 vezes) e o The Foos (4 vezes).

\section{Considerações Finais}

Com o objetivo de investigar o panorama brasileiro referente ao uso de ferramentas tecnológicas que contribuem para o desenvolvimento do Pensamento Computacional e para o ensino de programação de computadores, foi feita uma análise nos principais fóruns, promovidos pela SBC, que tratam sobre o tema no país.

Observou-se certa tendência dos estudos, cerca de 69,3\%, estarem direcionado aos níveis Fundamental, Superior e Médio, sinalizando uma certa carência deste tipo de pesquisas direcionadas ao nível Técnico e à Educação Infantil. Outra evidência é que mais da metade desses artigos utiliza alguma ferramenta tecnológica já existente, abrindo espaço para estudos que avaliem e/ou criem ferramentas direcionadas para o cenário nacional.

Outra questão que surgiu foi até que ponto essas ferramentas são realmente conhecidas e utilizadas pelos professores na sala de aula. Na sua maioria, os artigos apresentam estudos que foram feitos por meio de oficinas e, geralmente, realizadas no turno inverso da aula dos alunos participantes e sem o acompanhamento de seus professores. São pesquisas realizadas no meio acadêmico por estudantes de graduação e pós-graduação que vão às escolas apresentar essas ferramentas como parte de suas pesquisas, não ficando claro o retorno dessas pesquisas para o ambiente escolar. Percebese, então, a necessidade de se investigar, também, o que realmente estes professores conhecem deste conjunto de ferramentas e como podem usufruir desse arsenal. E, por fim, faz-se necessária uma análise mais direcionada às necessidades de alunos e professores relacionadas ao design destas ferramentas, incluindo dificuldades de interação e, principalmente, questões referentes ao contexto educacional brasileiro.

\section{Referências}

Amaral, E., Medina, R., Tarouco, L. M. R. (2016). Processo de ensino e aprendizagem de algoritmos integrando ambientes imersivos e o paradigma de blocos de programação visual. In: Anais dos Workshops do Congresso Brasileiro de Informática na Educação - SBIE, volume 5, page 20.

Andrade, D. et al. (2013). Proposta de atividades para o desenvolvimento do pensamento computacional no ensino fundamental. In: Anais do XXI Workshop de Informática na Escola - WEI, page 169. 
Avila, Christiano, et al. (2016). Desdobramentos do pensamento computacional no Brasil. In: Anais do Simpósio Brasileiro de Informática na Educação - SBIE, page 200.

Brackmann, C. P., Cassola, N. (2017). Desenvolvimento do pensamento computacional através de atividades desplugadas na educação básica. Disponível em:

https://www.ufrgs.br/ciencia/o-pensamento-computacional-no-ensino-fundamental/. Acesso em: 25 mar 2021.

Guarda, G. F, Pinto, S. C. (2020). Dimensões do Pensamento Computacional: conceitos, práticas e novas perspectivas. In: Anais do XXXI Simpósio Brasileiro de Informática na Educação - SBIE, pages 1463-1472.

ISTE. ISTE Standards for Students. (2016). International. Society for Technology in Education. Disponível em :https://www.iste.org/standards/for-students\#startstandards Acesso em: 25 mar 2021.

Kitchenham, B, Charters, Stuart. (2007) Guidelines for performing Systematic Literature Reviews in Software Engineering, Version 2.3, Technical Report - EBSE, Keele University and University of Durham.

Medina, M., Ferting, C. (2006) Algoritmos e programação: teoria e prática. São Paulo: Editora Novatec.

Nascimento, C., Santos, D. A., Tanzi, A. (2018) Pensamento computacional e interdisciplinaridade na educação básica: um mapeamento sistemático. In: Anais dos Workshops do Congresso Brasileiro de Informática na Educação - CBIE, page 709.

Ortiz, J. dos S. B.; Pereira, R. (2018). Um Mapeamento Sistemático Sobre as Iniciativas para Promover o Pensamento Computacional. In: Anais do XXXX Simpósio Brasileiro de Informática na Educação - SBIE), page 1093.

Papert, S. M. (1985) LOGO: Computadores e Educação. São Paulo, Editora Brasiliense, Tradução e prefácio de José A. Valente, da Unicamp, SP.

Polya, G. (1995). A arte de resolver problemas: um novo aspecto do método matemático. Tradução de Heitor Lisboa de Araújo. Rio de Janeiro: Interciência.

Zabot, D, Andrade, S., Matos, E. (2018). Raciocínio Computacional e Jogos Digitais: categorias e mecânicas. Anais do XXXX Simpósio Brasileiro de Informática na Educação - SBIE, page 76.

Zanetti, H. A. P.; Borges, M. A. F. ; Ricarte, I. L. M. (2016). Pensamento Computacional no Ensino de Programação: Uma Revisão Sistemática da Literatura Brasileira. In: Anais do XXXX Simpósio Brasileiro de Informática na Educação - SBIE, page 21.

Wing, J. M. (2006). Computational thinking. Communications of the ACM, volume 49, n. 3, pages 33-35. Disponível em: https://dl.acm.org/doi/10.1145/1118178.1118215 Acesso em: 25 mar 2021.

Wing, J. M. (2014). Computational Thinking Benefits Society. Social Issues in Computing. Academic Press New York. volume 2014, pages 26. Disponível em: $<$ http://socialissues.cs.toronto.edu/2014/01/computational-thinking/> . Acesso em: 25 mar 2021. 